\title{
An Egyptian HPAI H5N1 isolate from clade 2.2.1.2 is highly pathogenic in an experimentally infected domestic duck breed (Sudani duck)
}

\section{Short title: Egyptian HPAI H5N1 pathogenicity in domestic duck}

\author{
Mohamed Samir ${ }^{1,2^{*}}$, Marwa Hamed ${ }^{3}$, Fatma Abdallah ${ }^{4}$, Van Kinh Nguyen ${ }^{5,+}$, Esteban A. \\ Hernandez-Vargas ${ }^{5,+}$, Frauke Seehusen ${ }^{6}$, Wolfgang Baumgärtner ${ }^{6}$, Ashraf Hussein $^{7}$, Ahmed A. \\ H. Ali ${ }^{4}$ and Frank Pessler ${ }^{1,8^{*}}$
}

${ }^{1}$ TWINCORE, Center for Experimental and Clinical Infection Research, 30625, Hannover, Germany.

${ }^{2}$ Department of Zoonoses, Faculty of Veterinary Medicine, Zagazig University, 44511, Zagazig, Egypt.

${ }^{3}$ Animal Health Research Institute, Dokki, Giza, Egypt (Marsa matrouh branch).

${ }^{4}$ Department of Virology, Faculty of Veterinary Medicine, Zagazig University, 44511, Zagazig, Egypt.

${ }^{5}$ Systems Medicine of Infectious Diseases, Department of Systems Immunology and Braunschweig Integrated Centre of Systems Biology, Helmholtz Centre for Infection Research, Braunschweig, Germany.

${ }^{6}$ Department of Pathology, University of Veterinary Medicine, Hannover, Germany.

${ }^{7}$ Department of Avian and Rabbit Medicine, Faculty of Veterinary Medicine, Zagazig University, 44511, Zagazig, Egypt.

${ }^{8}$ Helmholtz Centre for Infection Research, Braunschweig, Germany.

${ }^{+}$Current affiliation: Frankfurt Institute for Advanced Studies, Frankfurt, Germany.

${ }^{*}$ Corresponding authors:

Frank Pessler, Email: frank.pessler@twincore.de Mohamed Samir, Email: mohsamir2016@yahoo.com / mshassan@zu.edu.eg Tel.: +49 511-220027-167

Fax: +49 511-220027-186 


\section{Abstract}

The highly pathogenic avian influenza (HPAl) H5N1 viruses continue to cause major problems in poultry and can, although rarely, cause human infection. Being enzootic in domestic poultry, Egyptian isolates are continuously evolving, and novel clades vary in their pathogenicity in avian hosts. Considering the importance of domestic ducks as natural hosts of HPAI H5N1 viruses and their likelihood of physical contact with other avian hosts and humans, it is of utmost importance to characterize the pathogenicity of newly emerged HPAI strains in the domestic duck. The most recently identified Egyptian clade 2.2.1.2 HPAI H5N1 viruses have been isolated from naturally infected pigeons, turkeys and humans. However, essentially nothing is known about their pathogenicity in domestic ducks. We therefore characterized the pathogenicity of an Egyptian HPAI H5N1 isolate A/chicken/Faquos/amn12/2011 (clade 2.2.1.2) in Sudani duck, a domestic duck breed commonly reared in Egypt. While viral transcription (HA mRNA) was highest in lung, heart and kidney peaking between 40 and $48 \mathrm{hpi}$, lower levels were detected in brain. Weight loss of infected ducks started16 hpi and persisted until $120 \mathrm{hpi}$. The first severe clinical signs were noted by $32 \mathrm{hpi}$ and peaked in severity at 72 and $96 \mathrm{hpi}$. Hematological analyses showed a decline in total leukocytes, granulocytes, platelets, and granulocyte/lymphocyte ratio, but lymphocytosis. Upon necropsy, lesions were obvious in heart, liver, spleen and pancreas and consisted mainly of necrosis and petechial hemorrhage. Histologically, lungs were the most severely affected organs, whereas brain only showed mild neuronal degeneration and gliosis at 48 hpi despite obvious neurological clinical signs. Taken together, our results provide first evidence that this HPAI H5N1 isolate (clade 2.2.1.2) is highly pathogenic to Sudani ducks and highlight the importance of this breed as potential reservoir and disseminator of HPAl strains from this clade.

\section{Keywords}

Domestic ducks, Egyptian H5N1 virus, experimental infection, highly pathogenic avian influenza (HPAl), kinetics, pathogenicity, clade 2.2.1.2 


\section{Introduction}

The highly pathogenic avian influenza (HPAI) viruses of the H5N1 lineage are of particular concern because they can cause systemic illness and high mortality in birds and also constitute zoonotic pathogens (Peiris et al., 2007). Although they can infect a broad range of domestic and wild birds (Alexander, 2000, Ramis et al., 2014), disease pathogenicity (Perkins and Swayne, 2002, Perkins and Swayne, 2001) and virusinduced immune responses (Burggraaf et al., 2014, Kuchipudi et al., 2014) differ among HPAI H5N1-infected bird species, which is also reflected in variations in the host response to vaccination (Cagle et al., 2011). Data from natural and experimental infections with HPAI H5N1 viruses revealed that chicken and turkey developed systemic disease followed by high mortality, whereas ducks showed minimal or no clinical signs despite being infected (Kuchipudi et al., 2014, Cornelissen et al., 2013, Kayali et al., 2011). Ducks shed high viral titers in pharyngeal and fecal excretion in the absence of debilitating disease, thus representing silent reservoirs and spreaders for HPAI H5N1 viruses (Webster et al., 1992, Kim et al., 2009). In late 2002, the ecology of HPAI H5N1 viruses in ducks changed in that many virus isolates became unusually lethal in this bird species (Sturm-Ramirez et al., 2004) as evidenced by reports from natural infection (e.g. in western China (Chen et al., 2005, Liu et al., 2005) and Korea (Rhyoo et al., 2015, Kwon et al., 2005)) as well as in experimental infections (Kishida et al., 2005, Yamamoto et al., 2007, Londt et al., 2008). This increased virulence of H5N1 in ducks has also been observed in Egyptian poultry (OIE, 2008). Two Egyptian H5N1 viruses isolated in 2007/2008, belonging to clade 2.2.1, were found to be highly pathogenic in Pekin ducks (Anas platyrhynchos) (Wasilenko et al., 2011). It has been suggested that the long persistence of the virus inside a natural host (e.g. ducks) after development of humeral immunity (Wibawa et al., 2014) and/or the extensive use of unapproved vaccines, as seen in a study done in Mexico (Lee et al., 2004), are responsible for such switch in virulence. Indeed, these factors impose immune pressure on the virus and drive mutations in its genome (Ludwig et al., 1995, Nguyen et al., 2017, Lee et al., 2016), leading to the appearance of genetically and antigenically novel strains. This increased virulence and the continuous evolution of 
HPAI H5N1 support the notion that ducks might be a major source of HPAI H5N1 outbreaks and pandemics.

The rearing system of poultry in Egypt has provided conditions in which HPAI H5N1 viruses underwent genetic and antigenic evolution (Watanabe et al., 2012, Sheta et al., 2014). As a result and in spite of vaccination efforts, multiple virus clades have been co-circulating in domestic poultry (Naguib et al., 2016). Historically, viruses within clade 2.2, which is thought to be the origin of the first Egyptian HPAI H5N1 isolate (Saad et al., 2007), quickly diversified and generated a so called classic clade (clade 2.2.1) (Abdelwhab et al., 2010), which was common in backyard and house hold poultry. Two main subgroups originated from this clade, (1) the variant group (clades 2.2.1.1/2.2.1.1a), which co-circulated mostly in vaccinated flocks between 2009-2011 and has not been detected since (WHO/OIE/FAOH. N. Evolution Working Group WHO, 2012), and more recently, (2) the endemic clade 2.2.1.2 (El-Shesheny et al., 2014, Smith et al., 2015), which was found to contain another distinct subclade (Arafa et al., 2015) that was later subdivided into A, B and C subclades (Salaheldin et al., 2017). Viruses within clade 2.2.1.2 have been isolated from domestic birds (e.g. turkey (Salaheldin et al., 2017) and pigeon (Mansour et al., 2017)) and from humans during the winter of 2014/2015 (El-Shesheny et al., 2017, Arafa et al., 2015). From 20092014, this clade represented the dominant circulating HPAI H5N1 isolate in households and commercial poultry irrespective of their vaccination status. Although viruses within clade 2.2.1.2 isolates have been reported to naturally infect ducks (Arafa et al., 2015), no reports exist that describe the details of their pathogenicity in locally raised domestic duck breeds in Egypt such as Sudani ducks. Indeed, understanding virus pathogenicity in such locally raised breeds is crucial due to many reasons. From a viral point of view, this clade has an Egypt-wide distribution (i.e. in upper and lower Egypt) with its HA gene being subject to high positive selection pressure, which facilitates viral adaptation to both environment and the avian host. Additionally, isolates within this clade exhibited a higher evolution rate $\left(6.9 \times 10^{-3}\right.$ substitution/site/year) and higher number of vaccine escape mutations than their ancestral viruses (Arafa et al., 2016). It

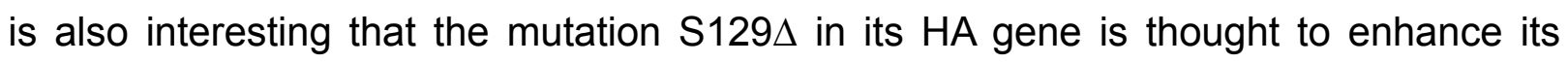
binding to the human-type receptor (Watanabe et al., 2011, Arafa et al., 2016). From a 
host perspective, the Sudani duck breed, a derivative of the Muscovy duck (Cairina moschata), is a locally reared breed that has become popular among Egyptian farmers and consumers due to its relatively low price and heat tolerance, compared to other breeds (Laila Abd El-Samee, 2012). This duck breed is mostly reared in backyards, either alone or mixed with other domestic poultry and/or sold in live poultry markets, thus representing a potential reservoir for HPAI H5N1 viruses (Kim et al., 2009) and might be implicated in future outbreaks in Egypt (Hassan et al., 2013, Wasilenko et al., 2011). Our previous experiments showed that Sudani ducks ( $n=3$ per time point) experimentally infected with $\mathrm{A} /$ chicken/Faquos/amn12/2011 were able to shed the virus, as determined by cloacal ( $10^{2}$ and $10^{1.8}$ EID $_{50}$ at 48 and $120 \mathrm{hpi}$, respectively) and nasal swabs ( $10^{4.9}$ and $10^{4.3} \mathrm{EID}_{50}$ at 48 and $120 \mathrm{hpi}$, respectively) (Salem, 2014a), demonstrating the potential of this breed to disseminate the virus to the environment and possibly other susceptible avian contacts. Knowledge about the in vivo kinetics of the virus, virus-associated lesions and correlating these to the clinical and histopathological indices of disease severity are important to understand pathogenesis, in particular any causes of the apparent high mortality. This relates directly to the interspecies transmission of the virus and subsequently may help design disease control and prevention strategies.

In the current study, we investigated the pathogenicity of the Egyptian HPAI H5N1 isolate named as $\mathrm{A} /$ chicken/Faquos/amn12/2011 (which has been assigned to the recently identified clade 2.2.1.2) in the Sudani duck breed as potential host. Our results indicate that Sudani ducks are highly susceptible to infection with this strain as evidenced by systemic viral spread, progressive development of clinical signs and symptoms, progressive hematological abnormalities, multi-organ lesions predominantly affecting the lung, and high mortality. The results demonstrate, for the first time, that an H5N1 virus isolate belonging to clade 2.2.1.2 is highly pathogenic in Sudani duck. This information, together with the ability of these ducks to shed the virus into the environment, suggest that Sudani duck should be considered when tracing the source of local HPAI H5N1 outbreaks in Egypt, particularly in rural areas, and when selecting and implementing outbreak and epidemic interventions. 


\section{Materials and methods}

\section{Virus propagation and titration}

We characterized the HPAI strain A/chicken/Faquos/amn12/2011(H5N1), accession number “JQ627585.1" (kindly provided by Dr. Abdo Nagy, Virology Department, Faculty of Veterinary Medicine, Zagazig University). The virus was isolated from following a fulminant outbreak in household broiler chicken flocks in Faquos territory, El-Sharkia province, Egypt. Procedures for virus propagation, titration and re-isolation were carried out as described previously (Salem, 2014b). Briefly, $200 \mu$ l of virus-PBS suspension were inoculated into 11-day-old specific pathogen-free (SPF) embryonated chicken eggs (SPF-ECEs), and equal numbers of SPF eggs were inoculated with an equal volume of PBS and were used as control group. Eggs were incubated at $37^{\circ} \mathrm{C}$ and $60 \%-70 \%$ humidity and monitored daily for viability. Non-viable eggs were collected and refrigerated at $4^{\circ} \mathrm{C}$. The propagated virus was identified in the harvested allantoic fluid by hemagglutination inhibition (HAI) test as described previously (Salem, 2014b). Virus titers were calculated according to the Reed and Muench method (REED and MUENCH, 1938).

\section{Phylogenetic and amino acid (aa) analyses}

In a previous study, we partially sequenced the HA mRNA of this isolate (Ahmed AbdelSamee Salama, 2012). According to the United States National Institutes of Allergic and Infectious Diseases (NIAID) Influenza Research Database, the virus was meanwhile assigned to clade 2.2.1.2 (Zhang et al., 2017). For confirmatory reasons, we carried out an independent phylogenetic analysis and protein alignment using the partial HA sequences available at NCBI (accession number: JQ627585.1). We ran the phylogenetic analysis after compiling the nucleotide sequence of the (A/chicken/Faquos/amn12/2011(H5N1)) HA gene together with $37 \mathrm{H} 5 \mathrm{~N} 1$ isolates that were isolated between 2006-2011 from different localities in Egypt and belong to various clades: 2.2, 2.2.1 (classic), 2.2.1.1 (variant) and 2.2.1.2. The included isolates and the corresponding accession numbers are summarized in Table S1. We also 
included the parental H5N1 virus that was isolated from geese in 1996 in Guangdong province. Evolutionary analyses were conducted using the Bioedit sequence alignment editor and the MEGA5 software tools (Tamura et al., 2011, Hall, 1999). The evolutionary history of the virus was inferred using the neighbor-joining method (Saitou and Nei, 1987) and the optimal tree with the sum of branch lengths $=0.44213287$ is shown (Figure 1). The percentage of replicate trees in which the associated taxa clustered together in the bootstrap test (1000 replicates) are shown next to the branches (Felsenstein, 1985). The evolutionary distances were computed using the $p$ distance method $(S, 2000)$ and are in the units of the number of base differences per site. All positions containing gaps and missing data were eliminated. There were 379 positions in the final dataset. For confirmation, we performed a protein analysis using a stretch of aa (110-165) of our isolate was compared to that of 27 other H5N1 isolates spanning the same clades that comprised the phylogenetic analysis (Table S2 and Figure S1).

\section{Animal experiment}

Two-week-old Sudani ducks were used. The ducks were housed in a sterilized selfcontained isolation unit under continuous lighting for 5 days. Prior to virus inoculation, hemagglutination inhibition ( $\mathrm{HAl}$ ) and enzyme-linked immunosorbant assay (ELISA) tests were applied on blood samples taken from all ducks to ensure the absence of previous H5N1 infection (Salem, 2014b). Ducks (average weight $730 \mathrm{~g}$ ) were separated into mock treatment $(n=30)$ and HPAI H5N1 infection groups $(n=60)$. The mock and the infection groups were inoculated intranasally with $100 \mu \mathrm{l}$ of PBS and 100 $\mu \mathrm{l}$ of a virus suspension containing $10^{6.7}$ embryonated egg infective dose 50 (EID $\left.D_{50}\right)$, respectively. Three ducks from each group were weighed in parallel at each time point. At least three infected ducks that showed clinical signs were euthanized at 8, 16, 24, 32, 40, 48, 72, 96 and 120 hours post infection (hpi). To calculate the cumulative survival rate, survival data were collected on a subgroup of 27 ducks throughout the time course. The animals were monitored daily for clinical signs, which were scored as follows: - = no signs, $+=$ nonspecific signs (e.g. ruffled feathers, nasal discharge and 
cloudy eyes), $++=$ specific signs (respiratory distress and labored breathing), and +++ = severe signs (e.g. neurological signs, incoordination and paralysis). Tissue from lung, intestine and brain was processed for histopathology (see below). The RNA-based analyses were not performed on intestine (due to poor RNA quality) and cecal tonsil, bursa of Fabricius, and liver (to limit the scope of the study). Post mortem changes in internal organs were recorded in ducks that had to be euthanized owing to the appearance of severe clinical signs (e.g. high degree of paralyses and severe neurological manifestations). All experimental work was performed under high biosafety conditions that comply with the local animal welfare regulation on experiments with ducks and similar species. The study was approved ahead by the Ethics Committee for Animal Studies at the Faculty of Veterinary Medicine, Zagazig University, Egypt.

\section{Hematological measurements}

Whole blood was collected from infected and mock-treated ducks (3 ducks from each group per time point) in tubes containing EDTA as anticoagulant, then analyzed with a SYSMEX XT-2000iv automated hematology analyzer (IDEXX Bio Research, 1-5-1, Wakinohama-Kaigandori, Chuo-ku, Kobe 651-0073, Japan) using duck as a background species according to the manufacturer's instructions. The following peripheral blood cells were measured: total leukocyte count (white blood cells, WBCs, $10^{3}$ per $\left.\mu \mathrm{l}\right)$, and absolute counts $\left(10^{3}\right.$ per $\left.\mu \mathrm{l}\right)$ and percentages of total granulocytes, heterophilic granulocytes, lymphocytes, monocytes, reticulocytes, the absolute platelet count $\left(10^{3}\right.$ per $\left.\mu \mathrm{l}\right)$ and granulocyte/lymphocyte ratio (absolute count of granulocyte/absolute count of lymphocyte). Student's $t$ test (two-tailed, heteroschedastic unpaired) was used to compare differences of mean values from H5N1-infected and mock-treated ducks at the same time point, using GraphPad Prism V.5 software (GraphPad Software, Inc., San Diego, California). 


\section{RNA extraction and reverse transcription}

Approx. $30 \mathrm{mg}$ of tissue was homogenized in a mixture of $600 \mu \mathrm{l}$ RLT buffer and $6 \mu \mathrm{l} \beta$ mercaptoethanol $(\beta-\mathrm{ME})$ using beads in a rotor-based homogenizer. Total RNA was extracted using the miRNeasy kit (Qiagen) and was then DNase-treated using the RNase-Free DNase Set (Qiagen). RNA quality and quantity were determined with the Nanodrop S1000 spectrophotometer (Thermo Scientific). The DNase-treated RNA was reverse transcribed using the miScript Reverse Transcription Kit (Qiagen) in a volume of $20 \mu \mathrm{l}$.

\section{Quantitative reverse transcriptase real-time PCR (RT-qPCR) and statistical analyses}

To measure H5N1 HA mRNA expression, RT-qPCR was performed using the miScript SYBR Green PCR Kit (Qiagen) and a Light Cycler 480 real-time PCR instrument (Roche, software version 1.5). Primer sets for H5N1 HA mRNA and duck $\beta$-actin mRNA were designed and checked for specificity based on melting curve analysis and gel electrophoresis of amplification products. The following primers were used (H5N1 HA: forward CAGCATGTCCATACCAGGGAA, reverse CCACTTTGCCCGTTTACCTT; $\beta$ actin: forward CCATTGAACACGGTATTGTCACC, reverse GCTACATACATGGCTGGGGT). To verify efficiency of the RT-qPCR reaction, two-fold serially diluted cDNA samples from kidney, lung, brain and heart were used to create a standard curve. Slope and correlation coefficient were calculated. The efficiencies ranged from $100-110 \%$, which corresponded to a range in slope from -3.49 to -3.1 . The cycling conditions started with pre-incubation at $95^{\circ} \mathrm{C}$ for $15 \mathrm{~min}$, followed by 40 amplification cycles at $94^{\circ} \mathrm{C}$ for 15 seconds (s), $55^{\circ} \mathrm{C}$ for $30 \mathrm{~s}$ and $72^{\circ} \mathrm{C}$ for $30 \mathrm{~s}$. Melting curves were generated by heating the samples to $95^{\circ} \mathrm{C}$. Fold change (FC) in expression was calculated using the $2^{-\Delta \Delta} C_{T}$ method (Livak and Schmittgen, 2001). One way ANOVA followed by Tukey's multiple comparisons test was used to compare differences in mean values, using graph pad prism V.5 software (GraphPad Software, Inc., San Diego, California). The RT-qPCR using the $\Delta \mathrm{Ct}$ values (HA mRNA 
normalized against $\beta$-actin mRNA) data were used to calculate exponential growth rates ( $g$ value) of the virus among the various organs. For this purpose, we made the assumption that the virus grows exponentially with rate g, i.e., $y_{t}=a e^{i}$, where $y$ is the viral concentration measured, $t$ is the corresponding time points, and $a$ is the intercept of the statistical model (Smith et al., 2010). In addition, Pearson correlation coefficient was used to measure correlations in viral transcription across the organs.

\section{Histopathological lesions in infected organs}

Tissue pieces were dissected from lung (random selection from right or left lung), brain (cerebral cortex) and small intestine (random selection from different parts) and then fixed in formalin for a maximum of $48 \mathrm{~h}$. The formalin-fixed tissues from at least 3 ducks per time point were processed according to standard procedures and embedded in paraffin. A triplicate of paraffin-embedded tissues were cut to a thickness of $3 \mu \mathrm{m}$, stained with hematoxylin/eosin (H\&E) and evaluated by one of the authors (FS), a board-certified veterinary pathologist.

\section{Results}

\section{Phylogenetic analysis and amino acid sequence characterization of the HA segment}

To confirm the genetic relatedness of the isolate studied, phylogenetic tree and amino acid (aa) analyses were carried out using the available HA mRNA sequences. Based on mRNA sequence, $A /$ chicken/Faquos/amn12/2011 isolate clustered with the recently emerged H5N1 clade 2.2.1.2., but was distinct from other and previously prevailing clades (Figure 1). For aa-based phylogeny, we aligned a sequence of 55 aa of the HA protein to the corresponding segment of a panel of HPAI H5N1 viruses belonging to clades that have been circulating among Egyptian poultry. This analysis agreed with the RNA-based phylogeny in that the study isolate shares a common aa signature with viruses belonging to clade 2.2.1.2 and that these residues differ from those of other 
H5N1 virus clades (Figure S1 and Table S2). These signatures are summarized in Table S2 and include H110, D120, S123, 1129 , R140, S141, F144, T151, N154, A156, K162 and N165. The study isolate shared some aa residues with viruses within clade 2.2 (e.g. H110, S123, R140, S141, F 144, A156 and N165). Nevertheless, differences between these clades were also noted (e.g. S120D, S129A, I151T, D154N and $\mathrm{R} 162 \mathrm{~K})$. Of note, the presence of D120 discriminated the clade 2.2.1.2, including our isolate, from all other clades. Some aa residues discriminated our virus from those

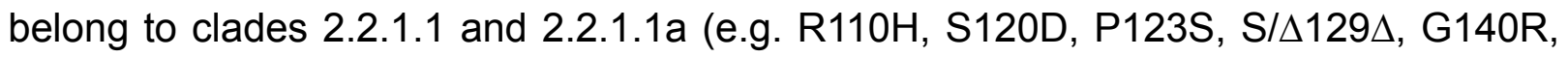
P141S, Y144F, and H165N).

\section{Clinical observations and findings of gross pathology}

The mock-treated ducks did not develop clinical signs, did not lose weight throughout the time course (Figure $2 \mathrm{~A}$ ), and gross pathological lesions were not seen upon necropsy. In the infected ducks, weight loss started after $16 \mathrm{hpi}$ and continued throughout the time course (Figure 2 A). The clinical scores are summarized in Table 1. During the first $24 \mathrm{hpi}$, the infected ducks showed general signs of illness including ruffled feathers, recumbency and decrease in food and water intake. At $32 \mathrm{hpi}$, two ducks demonstrated respiratory signs (e.g. labored breathing and dyspnea) and a third one showed neurological signs and died (Figure 2 B and Table 1). Thus, the first death was recorded at this time point. By $72 \mathrm{hpi}$, several ducks showed neurological manifestations (tremors, circling and loss of balance) followed by death (Table 1 and Figure 2 B). Pronounced neurological manifestations were also evident at the subsequent time points (Table 1). Morphological examination of the duck that died at 32 hpi revealed congestion and yellowish fluid in the abdominal cavity (Figure $\mathbf{3} \mathbf{A}$ ) and several areas of submucosal hemorrhage in the liver (Figure $3 \mathrm{~B}$ ). At $48 \mathrm{hpi}$, pancreas of infected ducks exhibited petechial hemorrhages (Figure $3 \mathrm{C}$ ). At $72 \mathrm{hpi}$, hearts of infected ducks showed multifocal necrosis (Figure $3 \mathrm{D}$ ). Swelling of the spleen was also a frequent finding. Later in infection (at $96 \mathrm{hpi}$ ), the few surviving ducks appeared severely dehydrated. 


\section{Histopathological lesions in selected ducks organs}

The histopathological analysis revealed that lung lesions were most prominent at 16 , 48 and 72 hpi. Lungs showed a lympho-histiocytic interstitial pneumonia and various degrees of dispersed necro-suppurative foci starting at $8 \mathrm{hpi}$ and becoming more pronounced at 16 (Figure $4 \mathrm{~A}$ ), 48 and $72 \mathrm{hpi}$. There was mild-to-moderate multifocal necrosis of epithelial cells. Additionally, hyperplasia of the airway-associated lymphoid tissue and moderate-to-severe hyperemia were noticed. In the brain, the lesions were much less severe than in the lung in that clearly pathological lesions such as neuronal degeneration and gliosis were observed in only one animal at 48 hpi (Figure 4 B). Small intestine showed mild-to-moderate lympho-plasmacytic to heterophilic inflammation. Lesions were more pronounced at 72 and 96 hpi (Figure 4 C). Mild abnormalities were also seen in lung of a small number of mock-treated ducks, e.g., some pulmonary hyperemia (Figure $4 \mathrm{D}$ ), whereas brain and intestine from mocktreated ducks were normal in appearance (Figures $4 \mathrm{E}$ and F).

\section{Changes in peripheral blood cell populations}

As shown in Figure 5, the measured blood parameters were stable in the mock-treated (uninfected) animals, as they were kept under physiologically stable conditions. This finding underscores the robustness of hematological measurements applied herein. In contrast, infection led to a general decline in numbers and percentages of WBCs, platelets, monocytes, total granulocytes, heterophilic granulocytes and the granulocyte/lymphocyte ratio. The WBCs started to declin at $40 \mathrm{hpi}$ and began to normalize (although not fully) through $120 \mathrm{hpi}$. Interestingly, platelet counts showed a significant decrease at all time points starting $8 \mathrm{hpi}$, but then mounted a full recovery by $120 \mathrm{hpi}$. In contrast, a rise of lymphocytes began as early as $8 \mathrm{hpi}$, was interrupted briefly at $40 \mathrm{~h}$, and then persisted through 72 and 96 hpi. Reticulocytes showed a significant increase at 16, 32, $72 \mathrm{hpi}$. The $40 \mathrm{~h}$ time point represented an exception in that, although not statistically significant, there was a tendency for decreased 
lymphocyte counts and increased granulocyte, heterophil counts. The Granulocyte/lymphocyte ratio was $<1$ except at $40 \mathrm{hpi}$ but close to 4 at $120 \mathrm{hpi}$, consistent with an overall trend toward normalization in blood parameters in the surviving ducks this late in infection. It is not clear whether the transient drop in lymphocytes and rise in granulocytes is due to the natural biology of the hematological response to HPAl infection in this duck species, or evidence of variation in hematological responses in individual ducks.

\section{Kinetics of viral transcription (HA mRNA) in selected duck organs}

As shown in Figure 6, HA mRNA was detected in all organs examined. Peak transcription was clearly highest in spleen, heart and kidney, followed by lung, and was lowest in brain. Kinetics of viral transcription varied markedly depending on the organ and the time post infection. They were similar in lung, trachea, heart, kidney, pancreas and spleen, where HA mRNA was first detected at $8 \mathrm{hpi}$, peaked by $48 \mathrm{hpi}$, and then decreased gradually. In most of the organs, HA mRNA expression had begun to decrease by 96 hpi. Interestingly, a short viral rebound could be observed in most of the organs by $120 \mathrm{hpi}$. In brain, viral kinetics differed in that HA mRNA could also be detected by $8 \mathrm{hpi}$, but then rose moderately to a sustained plateau for most of the remainder of the time course. Of note, some variations in HA expression were also observed in pancreas, spleen and kidney. As expected, HA mRNA was not detected in the mock-treated animals. Viral exponential transcription rate $(g)$ differed among the organs: during the first $48 \mathrm{hpi}$, it was highest in heart followed by spleen, lung, kidney, and trachea (range of $g=0.16-0.28)$, and lowest in pancreas and brain $(g<0.1)$ (Figure 6). With regard to correlations of HA transcription among these organs, high correlations were observed between lung vs spleen, kidney and trachea; between trachea vs. kidney, heart, and spleen; between brain vs. kidney. Low correlations were observed between lung vs brain, heart and pancreas, and between pancreas and kidney (Figure 7). 


\section{Discussion}

In this study, we assessed the pathogenicity of an Egyptian HPAI H5N1 isolate that belongs to clade 2.2.1.2 in a domestic duck breed (Sudani duck) in a time series experiment spanning 5 days. The ducks were highly susceptible to infection manifesting systemic multi-organ spread characterized by marked variation among organs in viral transcription rate and virus-induced tissue lesions. Although virus transcription rate and induced lesions were minimal in duck brain, the infected ducks showed clear neurological signs that started as early as $32 \mathrm{hpi}$. Taken together, the data suggest high pathogenicity of this isolate from HPAI H5N1 clade 2.2.1.2, and likely other viruses within this clade in Sudani ducks, and underscore their importance as origins or intermediates in future HPAl outbreaks in Egypt.

\section{Phylogenetic analysis}

The HPAI H5N1, which was introduced into Egypt in 2006 (Saad et al., 2007), has passed through several genetic diversification events (WHO/OIE/FAOH. N. Evolution Working Group WHO, 2012, Abdelwhab et al., 2010, El-Shesheny et al., 2014, Ibrahim et al., 2013), which led to the existence of heterogeneous co-circulating clades and subclades in Egyptian poultry. These viruses varied in nucleotide, aa sequences and evolutionary rate (Watanabe et al., 2012, Arafa et al., 2016), which was proven, at least for some strains, to impact their differential pathogenicity in domestic Pekin ducks (Wasilenko et al., 2011). The phylogenetic tree and aa analysis performed here further confirm the genetic relatedness of the studied isolate to clade 2.2.1.2. and agree well with published sequences of other isolates belonging to this clade (WHO/OIE/FAOH. N. Evolution Working Group WHO, 2012, El-Shesheny et al., 2014). It has been previously thought that $60 \%$ of the viruses that belong to clade 2.2.1.2 have four mutations at the same site (i.e. S120D, I151T, D154N and R162K), which we also identified in our analyses (Arafa et al., 2016) as shown in Table S2 and Figure S1. These aa changes might affect the anti-viral immune response and thus the response 
to vaccination (Hoffmann et al., 2005). Of note, the deletion at site 129 that we identified in the current analyses is unique to A/chicken/Faquos/amn12/2011(H5N1) virus as well as the other isolates within clade 2.2.1.2. This mutation is located in the receptor binding domain (RBD) of the HA gene of viruses within 2.2.1.2 clade that were isolated from various bird species (e.g. chicken, ducks, turkey, geese and ostrich), the majority of human isolates in Egypt in 2009 and all H5N1 viruses isolated afterwards (Watanabe et al., 2011). This mutation has been linked to emergence of this clade in 2008 (Arafa et al., 2016) and was shown to increase attachment to and infectivity of human lower respiratory tract, particularly if combined with the I151T mutation, which we also observed in the current study (Watanabe et al., 2011). This suggest the high zoonotic risk posed by these viruses. The presence of the S120D mutation uniquely in our isolate and other isolates from clade 2.2.1.2 suggests that this residue may represent a central feature of this clade. It is worth noting that the T156A mutation, which was observed in our analysis and was also reported by El-Shesheny et al., might lead to a change in the glycosylation site at positions 154 - 156, which may further increase the likelihood of transmission of clade 2.2.1.2 isolates to humans (Herfst et al., 2012). Taken together, this analysis highlights the need for continuous surveillance for HPAI H5N1 isolates in order to correctly assign them to specific clades and to predict their potential impact on poultry and human health.

Correlates of clinical severity of infection with the study isolate.

In the current study, we showed that the Sudani duck is highly susceptible to infection with the HPAI A/chicken/Faquos/amn12/2011(H5N1) isolate, as evidenced by pronounced clinical signs and high mortality. The virus disseminated systemically and reached most of the internal organs, including the brain, although to a lesser extent. Of note, the virus was detected in brain of ducks with neurological signs, although at a markedly lower level than in other organs (Figure 6). Moreover, histological changes in brain were absent or low grade. This finding needs to be interpreted with caution because only cerebral cortex was examined histologically. Thus, it remains to be studied whether the high degree of neurological signs is due to direct viral effects upon the CNS, particularly infection of deeper parts of the brain that were not examined in 
the present study, or due to an encephalopathy resulting from systemic inflammation or metabolic dysfunction due to involvement of internal organs. The clinical signs observed in the current study agree with that of previous reports. For instance, HPAI H5N1 viruses within clade 2.2.1 isolated in 2007 and 2008 were pathogenic to Pekin ducks causing various clinical signs such as dyspnea and nervous signs (Wasilenko et al., 2011). Muscovy and Pekin ducks succumbed to the infection with HPAI H5N1 clades 2.2.1.1 and 2.2.1 even when various inoculation routes were used (PantinJackwood et al., 2013). However, it should be noted that viruses within the same clade may differ in virulence in domestic ducks (Wasilenko et al., 2011) and it would therefore be important to assess the pathogenicity of other clade 2.2.1.2 isolates in Sudani ducks in order to assess the generalizability of our findings.

\section{Histological correlates of systemic spread of infection}

The histological analyses revealed that the lung clearly was the most severely affected organ. Similar results have been described by others, albeit in different duck breeds (purebred mallard and Peking) and after using various inoculation routes (intranasal, intraocular and oral) (Londt et al., 2008, Wasilenko et al., 2011, Keawcharoen et al., 2008). Lungs were much more affected than brain at the tissue level (Figure $4 \mathbf{A}$ and B), which correlates well with the differences of viral HA mRNA levels and transcription rates in these organs (Figure 6). The virus-induced changes in lung tissue apparently preceded, with a certain delay, the appearance of signs of respiratory distress. For instance, interstitial pneumonia manifested by $16 \mathrm{hpi}$, whereas the respiratory symptoms began to appear at 32 hpi (Figure 4 and Table 1). Data from previous studies revealed that HPAI H5N1 virus can severely affect multiple organs, likely because of its high virulence (Londt et al., 2008, Vascellari et al., 2007). In contrast, this multi-organ involvement of HPAI (H5N1) infection is not seen in infection with low pathogenic avian influenza (LPAl) viruses (Daoust et al., 2011). This differential involvement of internal organs suggests that, as part of an outbreak investigation, veterinarians might need to investigate internal organs in order to assess pathogenicity of the causative strain early on. 
Reprogramming of blood cells after H5N1 infection in ducks

Monitoring blood cell counts after IAV infection has been studied in details in mouse models of influenza virus infection (Preusse et al., 2015, Dengler et al., 2014, Preusse et al., 2017) and provided valuable information as to the association of changes in blood parameters to disease severity. However, this far only one study (Mahmoud, 2015) that described hematological changes in ducks naturally infected with HPAI H5N1 virus. Our results with regard to the decrease in total WBCs, heterophils and monocytes (Figure 5 A, F and D) largely agree with that study (Mahmoud, 2015). The decreasing platelet counts might be due to depression of thrombocytopoiesis during virus systemic spread (Coles, 1986), sequestration in the enlarged spleen or formation of thrombi in organs not examined histologically in this study, and it might have contributed to the observed hemorrhage and congestion in the internal organs particularly at $32 \mathrm{hpi}$ (Figure $3 \mathrm{~A}$ ). The decrease in total WBC count, which manifested in the later stage of infection (Figure $5 \mathbf{A}$ ), clearly suggests the importance of leukopenia as an indication of H5N1 infection in Sudani ducks. In addition, a robust thrombocytopenia was observed which corrected with clinical recovery in the surviving animals. In our study, lymphocytosis was evident at all time points except for $40 \mathrm{hpi}$, which contrasts the results published by Essam et al. (Mahmoud, 2015). Indeed, we observed a characteristic picture in the blood parameters at this time point. As shown in Figure 5 A, C, E, F and H, WBC count and lymphocytes declined at $40 \mathrm{hpi}$, whereas granulocytes, heterophils and granulocyte/lymphocyte ratio showed the reverse. Based on these results and correlating it to the virus mRNA peak in spleen and other organs (i.e. 40 - 48 hpi) (Figure 6), we hypothesize that the high viral load in the organs compromised synthesis of lymphocytes and other WBCs particularly at this time point. While our findings regarding lymphocytes and granulocytes (Figure 5 C and E) contrast previous reports in well-established mouse models experimentally infected with H1N1 influenza viruses (Dengler et al., 2014, Preusse et al., 2015, Preusse et al., 2017), they agree well with other reports in H5N1-naturally infected ducks (Mahmoud, 2015). We have shown previously that an increased granulocyte/lymphocyte ratio is a 
marker for severe IAV (H1N1) in mice (Preusse et al., 2015, Dengler et al., 2014, Preusse et al., 2017) but the current data suggest that this ratio may actually be generally decreased in H5N1 infection in ducks except at 40 and 120 hpi. H5N9 virus infection of chickens caused depletion in lymphocytes due to virus invasion into various lymphoid organs (Van Campen et al., 1989), but in the current study a lymphocytosis was observed. Reticulocytes were sporadically elevated, indicating some attempt of the hemopoietic system to increase production of erythrocytes throughout infection. Of note, the observed late rebound in viral HA mRNA transcription (i.e. at $120 \mathrm{hpi}$ ) in some organs (Figure 6) might be associated with increase in some blood parameters (e.g. heterophiles, reticulocytes and granulocyte/lymphocyte ratio) (Figure 5) at the same late time points. The pathophysiological reasons for these differences and association remain to be explained in further studied.

\section{Potential causes of severe disease and/or death}

The results presented here suggest that neurological dysfunction and multi-organ failure contributed greatly to mortality of infected ducks. The high virulence of HPAI H5N1 has previously been attributed at least in part to a high degree of CNS invasion (Kishida et al., 2005), as in the latter study titers of the more virulent strain (DK/Yokohama/03) exceeded those of the less virulent one (Ck/Yamaguchi/). This seems to be true in wild ducks (e.g. tufted ducks and pochards) as well, which were highly susceptible to HPAI H5N1 infection (Keawcharoen et al., 2008), indicating that H5N1 invasion into the CNS may represent a mutual feature across species. In a previous study that involves Pekin ducks challenged with HPAI H5N1 viruses clade 2.2.1 (Wasilenko et al., 2011), the more virulent virus caused brain lesions that are similar to the ones that we observed. However, the onset of neurological signs was earlier in our study (at 32 hpi vs 2.6 days in (Wasilenko et al., 2011)), suggesting that our virus, which is a descendant from clade 2.2.1, might have become more virulent in Sudani ducks. We observed viral transcription In duck heart, (Figure 6) and multiple necrosis was also observed in this organ upon post mortem examination (Figure $3 \mathrm{D}$ ), suggesting that cardiac dysfunction might have contributed to the observed mortalities. This agrees with previous reports in HPAI H5N1 naturally infected meat-type ducks 
(Rhyoo et al., 2015) and Pekin ducks experimentally infected with Egyptian HPAI H5N1 (Wasilenko et al., 2011). The pancreatic lesions found in this study (Figure 3 C) were also reported previously in ducks naturally infected with HPAI H5N1 virus (Rhyoo et al., 2015). Taken together, these data suggest that HPAI H5N1 virus spreads across multiple organs, leading to various combinations of multiorgan failure, which underlies the observed high mortality.

Kinetics of viral transcription and virus systemic spread

Although influenza A virus (IAV) kinetics have been investigated in various studies (Beauchemin and Handel, 2011, Hernandez-Vargas et al., 2014), the models generally did not explore viral kinetics in multiple compartments that may have different virus replication patterns and hence reflect the spread of the virus in various body tissues. Several mathematical models can be applied for this purpose, e.g. the target cell limited model and the logistic growth model (Baccam et al., 2006). When data on viral replication are restricted to HA mRNA expression, model parameter estimation is difficult to perform, and regression models and correlation studies could be more suitable to assist the interpretation of the experimental data, for instance by modeling of kinetic curves (Ramsay et al., 2007). Viral exponential transcription rates varied markedly among organs and HA mRNA expression declined notably after $48 \mathrm{hpi}$ (Figures 6). The inter-organ variability in virus transcription rates is a common observation in avian influenza virus infections. Possible factors accounting for this include differences among organs in receptor availability and spatial distribution (Kuchipudi et al., 2009), induction of immune-related components (Wei et al., 2013), and apoptosis (Cornelissen et al., 2013). Another possible reason might be the variation in the track taken by the virus after infection. For instance, after intranasal inoculation, as in our experiment, the virus possibly reaches the brain mainly through olfactory and trigeminal nerves as suggested previously (Bodewes et al., 2011). In this case, the virus might reach other internal organs via the bloodstream (Yen et al., 2009). In natural infection, ducks might contract HPAI H5N1 through more than one portal of entry (e.g., both nasal and oral) and host immunity is frequently compromised by a variety of factors such as poor hygiene, mixed breeding and co-infections. The decline 
in viral transcription in some organs suggests an induction of immune response and/or depletion in virus target cells (Pawelek et al., 2012). Similar findings have been reported in lung and trachea of ducks experimentally infected with other strains of H5N1 viruses (Kishida et al., 2005, Tang et al., 2009). Interestingly, in certain organs such as lung, trachea and heart, a short second peak of HA transcription was observed at 120 hpi. While there is no direct explanation for this in avian hosts, similar phenomena have been described for H4N8 and H4N6 viruses in mice (Bui et al., 2012) and in H5N1 virus clade 2.2 after single passage in mice and in cell lines (Mase et al., 2006, Bogs et al., 2011). Reversion mutation in virus PB2 protein (e.g. E627K) might lead to this late increase in virus transcription. Another study (Pawelek et al., 2012) has provided an explanation to this phenomenon during H3N8 infection in ponies. The authors reported that the gradual loss of IFN-induced antiviral effects at $48 \mathrm{hpi}$, resulting in a reversion of the host cell from a refractory to a susceptible state. This explanation is supported by the fact that virus-resistant cells cannot maintain resistance without continuous IFN signaling (Pawelek et al., 2012). It remains to be determined why this virus rebound was observed only in some organs. However, it is tempting to speculate that under natural conditions, where the bird is dehydrated and its immunity is suppressed, exposure to several attacks of the same H5N1 virus might produce similar second virus peaks involving more organs. The fluctuations in viral transcription that were seen in pancreas, kidney and spleen in our study (Figures 6) might be a result of host-virus interaction and host genetic background (Londt et al., 2008). The correlation analysis in Figure 7 showed strongest correlation in HA mRNA expression between lung vs. both spleen and kidney, indicating that viral replication in spleen and kidney most closely paralleled that in lung. The low correlation values in virus HA mRNA level between brain and other organs (Figure 7) support that the virus reachs brain through the olfactory and trigeminal nerve after the intransasl inoculation and not as an extension from other organs. Thus, for further experimental work, it is recommended to divide the virus inoculum into many routs. The current study indicated that an H5N1 virus from clade 2.2.1.2 could spread systemically in Sudani duck, producing variable degree of clinical signs and damage in internal organs. Thus, this duck breed can act as a reservoir of this virus. In addition our previous study had 
demonstrated high viral titers in cloacal and pharyngeal swabs, strongly suggesting that the Sudani duck can act as a disseminator of H5N1 viruses belonging to this clade, and possibly others.

\section{Limitations of the present study}

This study is limited by several factors. Firstly, to avoid post-mortem artefacts, all ducks found dead at the scheduled time points were excluded from RT-qPCR and histopathological analyses. Thus, we may have under-estimated the true extent of viral spread and the severity of histopathological changes. Secondly, HA mRNA expression could not be assessed in intestine due to low quality of the RNA isolated from this organ. Thirdly, due to local resource limitations at the time of the infection experiment, viral titers in the various organs by inoculation of chicken eggs could not be determined, and HA mRNA expression was therefore used as proxy for viral replication. Fourthly, we only analyzed samples from the cerebral cortex, but not from deeper brain tissues because we propose to find virus-related pathological lesions in this site.

\section{Conclusions}

This study describes, for the first time, the details of increased pathogenicity of an HPAI H5N1 isolate from the recently described clade 2.2.1.2 in a commonly reared Egyptian domestic duck breed (Sudani duck). The results represent an important extension on previous observations of increased pathogenicity of HPAI H5N1 virus in domestic ducks. Our data add Sudani ducks as potential reservoirs and disseminators of strains from this clade and underscore the importance of continuous and close investigation of this local breed in future HPAI H5N1 outbreaks.

\section{Acknowledgments}

This work was completed as part of Mohamed Samir's PhD dissertation at the University of Veterinary Medicine (TiHo), Hannover, Germany. The study was supported by a German Egyptian Research Long Term Scholarship (GERLS), a joint 
program between the German Academic Exchange Service (DAAD) and the Egyptian Ministry of Higher Education and Scientific Research (grant ID: A/11/92510) to M. Samir, by funds from Zagazig University, and by iMed, the Helmholtz Association's Initiative on Personalized Medicine. This work was also partially supported by the Niedersachsen-Research Network on Neuroinfectiology (N-RENNT) of the Ministry of Science and Culture of Lower Saxony, Germany. We would like to thank Dr. Mohammed Nooruzzaman (TWINCORE Center for Experimental and Clinical Infection Research, Hannover, Germany) for support with the phylogenetic analysis, Dr. Marina Pils and Anna Rinkel (Helmholtz Center for Infection Research, Braunschweig, Germany) for support with histology.

\section{Conflict of interest}

The authors declare that they have no conflict of interest relating to conduct of the study or publication of this manuscript. 


\section{References}

Abdelwhab, E. M., A. S. Arafa, A. M. Erfan, M. M. Aly and H. M. Hafez, 2010: Modified H5 real-time reverse transcriptase-PCR oligonucleotides for detection of divergent avian influenza H5N1 viruses in Egypt. Avian diseases, 54, 1301-1305.

Ahmed Abdel-Samee Salama, I., M.E.B., Fatma Abdalla. and Nagy,A.M., 2012: Isolation and Identification of Avian Influenza Virus from Broiler Flocks in Sharqyia Governorate. 2012 edn. Virology Department, Faculty of Veterinary, Medicine, Zagazig Univ, Egypt, El-Zeraa Street, Zagazig, Sharqyia, 44511, Egypt, PubMed (https://www.ncbi.nlm.nih.gov)

Alexander, D. J., 2000: A review of avian influenza in different bird species. Veterinary microbiology, 74, 3-13.

Arafa, A., I. El-Masry, S. Kholosy, M. K. Hassan, G. Dauphin, J. Lubroth and Y. J. Makonnen, 2016: Phylodynamics of avian influenza clade 2.2.1 H5N1 viruses in Egypt. Virol J., 13, 49.

Arafa, A. S., M. M. Naguib, C. Luttermann, A. A. Selim, W. H. Kilany, N. Hagag, A. Samy, A. Abdelhalim, M. K. Hassan, E. M. Abdelwhab, Y. Makonnen, G. Dauphin, J. Lubroth, T. C. Mettenleiter, M. Beer, C. Grund and T. C. Harder, 2015: Emergence of a novel cluster of influenza A(H5N1) virus clade 2.2.1.2 with putative human health impact in Egypt, 2014/15. Euro surveillance : bulletin Europeen sur les maladies transmissibles = European communicable disease bulletin, 20, 2-8.

Baccam, P., C. Beauchemin, C. A. Macken, F. G. Hayden and A. S. Perelson, 2006: Kinetics of influenza A virus infection in humans. Journal of virology, 80, 7590-7599.

Beauchemin, C. and A. Handel, 2011: A review of mathematical models of influenza A infections within a host or cell culture: lessons learned and challenges ahead. BMC Public Health, 11, S7.

Bodewes, R., J. H. C. M. Kreijtz, G. van Amerongen, R. A. M. Fouchier, A. D. M. E. Osterhaus, G. F. Rimmelzwaan and T. Kuiken, 2011: Pathogenesis of Influenza A/H5N1 Virus Infection in Ferrets Differs between Intranasal and Intratracheal Routes of Inoculation. The American Journal of Pathology, 179, 30-36.

Bogs, J., D. Kalthoff, J. Veits, S. Pavlova, M. Schwemmle, B. Manz, T. C. Mettenleiter and J. Stech, 2011: Reversion of PB2-627E to -627K during replication of an H5N1 Clade 2.2 virus in mammalian hosts depends on the origin of the nucleoprotein. Journal of virology, 85, 10691-10698.

Bui, V. N., H. Ogawa, Xininigen, K. Karibe, K. Matsuo, S. S. Awad, G. L. Minoungou, S. Yoden, H. Haneda, L. H. Ngo, S. Tamaki, Y. Yamamoto, K. Nakamura, K. Saito, Y. Watanabe, J. Runstadler, F. Huettmann, G. M. Happ and K. Imai, 2012: H4N8 subtype avian influenza virus isolated from shorebirds contains a unique PB1 gene and causes severe respiratory disease in mice. Virology, 423, 77-88.

Burggraaf, S., A. J. Karpala, J. Bingham, S. Lowther, P. Selleck, W. Kimpton and A. G. Bean, 2014: H5N1 infection causes rapid mortality and high cytokine levels in chickens compared to ducks. Virus research, 185, 23-31.

Cagle, C., T. L. To, T. Nguyen, J. Wasilenko, S. C. Adams, C. J. Cardona, E. Spackman, D. L. Suarez and M. J. Pantin-Jackwood, 2011: Pekin and Muscovy ducks respond differently to vaccination with a H5N1 highly pathogenic avian influenza (HPAl) commercial inactivated vaccine. Vaccine, 29, 6549-6557.

Chen, H., G. J. Smith, S. Y. Zhang, K. Qin, J. Wang, K. S. Li, R. G. Webster, J. S. Peiris and Y. Guan, 2005: Avian flu: H5N1 virus outbreak in migratory waterfowl. Nature, 436, 191-192.

Coles, E., 1986: Veterinary Clinical Pathology. W.B. Saunders Company, Philadelphia, London.

Cornelissen, J. B., L. Vervelde, J. Post and J. M. Rebel, 2013: Differences in highly pathogenic avian influenza viral pathogenesis and associated early inflammatory response in chickens and ducks. Avian pathology : journal of the W.V.P.A, 42, 347-364. 
Daoust, P. Y., F. S. Kibenge, R. A. Fouchier, M. W. van de Bildt, D. van Riel and T. Kuiken, 2011: Replication of low pathogenic avian influenza virus in naturally infected Mallard ducks (Anas platyrhynchos) causes no morphologic lesions. Journal of wildlife diseases, 47, 401-409.

Dengler, L., N. Kuhn, D. L. Shin, B. Hatesuer, K. Schughart and E. Wilk, 2014: Cellular changes in blood indicate severe respiratory disease during influenza infections in mice. PloS one, 9, e103149.

El-Shesheny, R., A. Kandeil, O. Bagato, A. M. Maatouq, Y. Moatasim, A. Rubrum, M. S. Song, R. J. Webby, M. A. Ali and G. Kayali, 2014: Molecular characterization of avian influenza H5N1 virus in Egypt and the emergence of a novel endemic subclade. The Journal of general virology, 95, 14441463.

El-Shesheny, R., A. Mostafa, A. Kandeil, S. H. Mahmoud, O. Bagato, A. Naguib, S. E. Refaey, R. J. Webby, M. A. Ali and G. Kayali, 2017: Biological characterization of highly pathogenic avian influenza H5N1 viruses that infected humans in Egypt in 2014-2015. 162, 687-700.

Felsenstein, J., 1985: Confidence limits on phylogenies: an approach using the bootstrap.

Hall, N., 1999: Bioedit: a user-friendly biological sequence alignment editor and analysis program for windows 95/98/NT. Nucl. Acids Symp, 41, 95-98.

Hassan, M. K., Y. Jobre, A. Arafa, E. M. Abdelwhab, W. H. Kilany, S. G. Khoulosy, N. R. Bakry, E. Baile, A. Ali, P. Ankers and J. Lubroth, 2013: Detection of A/H5N1 virus from asymptomatic native ducks in mid-summer in Egypt. Archives of virology, 158, 1361-1365.

Herfst, S., E. J. Schrauwen, M. Linster, S. Chutinimitkul, E. de Wit, V. J. Munster, E. M. Sorrell, T. M. Bestebroer, D. F. Burke, D. J. Smith, G. F. Rimmelzwaan, A. D. Osterhaus and R. A. Fouchier, 2012: Airborne transmission of influenza A/H5N1 virus between ferrets. Science (New York, N.Y.), 336, 1534-1541.

Hernandez-Vargas, E. A., E. Wilk, L. Canini, F. R. Toapanta, S. C. Binder, A. Uvarovskii, T. M. Ross, C. A. Guzman, A. S. Perelson and M. Meyer-Hermann, 2014: Effects of aging on influenza virus infection dynamics. Journal of virology, 88, 4123-4131.

Hoffmann, E., A. S. Lipatov, R. J. Webby, E. A. Govorkova and R. G. Webster, 2005: Role of specific hemagglutinin amino acids in the immunogenicity and protection of H5N1 influenza virus vaccines. Proc. Natl. Acad. Sci. U. S. A., 102, 12915-12920.

Huber, P. J., 1981: Robust Statistics. Wiley.

Ibrahim, M., A.-F. Eladl, H. A. Sultan, A. S. Arafa, A. G. Abdel Razik, S. Abd El Rahman, K. I. A. El-Azm, Y. M. Saif and C.-W. Lee, 2013: Antigenic analysis of H5N1 highly pathogenic avian influenza viruses circulating in Egypt (2006-2012). Vet. Microbiol., 167, 651-661.

Kayali, G., R. El-Shesheny, M. A. Kutkat, A. M. Kandeil, A. Mostafa, M. F. Ducatez, P. P. McKenzie, E. A. Govorkova, M. H. Nasraa, R. G. Webster, R. J. Webby and M. A. Ali, 2011: Continuing threat of influenza (H5N1) virus circulation in Egypt. Emerging infectious diseases, 17, 2306-2308.

Keawcharoen, J., D. van Riel, G. van Amerongen, T. Bestebroer, W. E. Beyer, R. van Lavieren, A. D. Osterhaus, R. A. Fouchier and T. Kuiken, 2008: Wild ducks as long-distance vectors of highly pathogenic avian influenza virus (H5N1). Emerging infectious diseases, 14, 600-607.

Kim, J. K., N. J. Negovetich, H. L. Forrest and R. G. Webster, 2009: Ducks: the "Trojan horses" of H5N1 influenza. Influenza and other respiratory viruses, 3, 121-128.

Kishida, N., Y. Sakoda, N. Isoda, K. Matsuda, M. Eto, Y. Sunaga, T. Umemura and H. Kida, 2005: Pathogenicity of H5 influenza viruses for ducks. Archives of virology, 150, 1383-1392.

Kuchipudi, S. V., R. Nelli, G. A. White, M. Bain, K. C. Chang and S. Dunham, 2009: Differences in influenza virus receptors in chickens and ducks: Implications for interspecies transmission. Journal of molecular and genetic medicine : an international journal of biomedical research, 3, 143-151.

Kuchipudi, S. V., M. Tellabati, S. Sebastian, B. Z. Londt, C. Jansen, L. Vervelde, S. M. Brookes, I. H. Brown, S. P. Dunham and K. C. Chang, 2014: Highly pathogenic avian influenza virus infection in 
chickens but not ducks is associated with elevated host immune and pro-inflammatory responses. Veterinary research, 45, 118.

Kwon, Y. K., S. J. Joh, M. C. Kim, H. W. Sung, Y. J. Lee, J. G. Choi, E. K. Lee and J. H. Kim, 2005: Highly pathogenic avian influenza (H5N1) in the commercial domestic ducks of South Korea. Avian pathology : journal of the W.V.P.A, 34, 367-370.

Laila Abd El-Samee, H. M. H. E.-A. a. N. A. M., 2012: Comparative Study on Some Productive Traits of Muscovy and Sudani Ducks in Egypt. International Journal of Poultry Science, 11, 264-268.

Lee, C. W., D. A. Senne and D. L. Suarez, 2004: Effect of vaccine use in the evolution of Mexican lineage H5N2 avian influenza virus. Journal of virology, 78, 8372-8381.

Lee, D. H., A. Fusaro, C. S. Song, D. L. Suarez and D. E. Swayne, 2016: Poultry vaccination directed evolution of H9N2 low pathogenicity avian influenza viruses in Korea. Virology, 488, 225-231.

Liu, J., H. Xiao, F. Lei, Q. Zhu, K. Qin, X. W. Zhang, X. L. Zhang, D. Zhao, G. Wang, Y. Feng, J. Ma, W. Liu, J. Wang and G. F. Gao, 2005: Highly pathogenic H5N1 influenza virus infection in migratory birds. Science (New York, N.Y.), 309, 1206.

Livak, K. J. and T. D. Schmittgen, 2001: Analysis of relative gene expression data using real-time quantitative PCR and the 2(-Delta Delta C(T)) Method. Methods (San Diego, Calif.), 25, 402-408.

Londt, B. Z., A. Nunez, J. Banks, H. Nili, L. K. Johnson and D. J. Alexander, 2008: Pathogenesis of highly pathogenic avian influenza A/turkey/Turkey/1/2005 H5N1 in Pekin ducks (Anas platyrhynchos) infected experimentally. Avian pathology : journal of the W.V.P.A, 37, 619-627.

Ludwig, S., L. Stitz, O. Planz, H. Van, W. M. Fitch and C. Scholtissek, 1995: European swine virus as a possible source for the next influenza pandemic? Virology, 212, 555-561.

Mahmoud, E. A., 2015: Hemato-biochemical and pathological changes on avian influenza in naturally infected domestic ducks in Egypt. Veterinary world, 8, 1177-1182.

Mansour, S. M., F. F. Mohamed, A. A. Eid, S. K. Mor and S. M. Goyal, 2017: Co-circulation of paramyxoand influenza viruses in pigeons in Egypt. Avian Pathol., 1-9.

Mase, M., N. Tanimura, T. Imada, M. Okamatsu, K. Tsukamoto and S. Yamaguchi, 2006: Recent H5N1 avian influenza $A$ virus increases rapidly in virulence to mice after a single passage in mice. The Journal of general virology, 87, 3655-3659.

Naguib, M. M., E. M. Abdelwhab and T. C. Harder, 2016: Evolutionary features of influenza A/H5N1 virus populations in Egypt: poultry and human health implications. Arch. Virol., 161, 1963-1967.

Nguyen, L. T., T. Nishi, S. Shichinohe, D. H. Chu, T. Hiono, K. Matsuno, M. Okamatsu, H. Kida and Y. Sakoda, 2017: Selection of antigenic variants of an H5N1 highly pathogenic avian influenza virus in vaccinated chickens. Virology, 510, 252-261.

OIE, 2008: OIE, Follow-up report, OIE Ref: 7012. http://www.oie.int/downld/AVIAN \%20INFLUENZA/Eqypt followup7 final\%20report.pdf.

Pantin-Jackwood, M., D. E. Swayne, D. Smith and E. Shepherd, 2013: Effect of species, breed and route of virus inoculation on the pathogenicity of H5N1 highly pathogenic influenza (HPAI) viruses in domestic ducks. Vet. Res., 44, 62.

Pawelek, K. A., G. T. Huynh, M. Quinlivan, A. Cullinane, L. Rong and A. S. Perelson, 2012: Modeling within-host dynamics of influenza virus infection including immune responses. PLOS computational biology, 8, e1002588.

Peiris, J. S., M. D. de Jong and Y. Guan, 2007: Avian influenza virus (H5N1): a threat to human health. Clinical microbiology reviews, 20, 243-267.

Perkins, L. E. and D. E. Swayne, 2001: Pathobiology of A/chicken/Hong Kong/220/97 (H5N1) avian influenza virus in seven gallinaceous species. Veterinary pathology, 38, 149-164.

Perkins, L. E. and D. E. Swayne, 2002: Pathogenicity of a Hong Kong-origin H5N1 highly pathogenic avian influenza virus for emus, geese, ducks, and pigeons. Avian Dis., 46, 53-63. 
Preusse, M., K. Schughart and F. Pessler, 2017: Host Genetic Background Strongly Affects Pulmonary microRNA Expression before and during Influenza A Virus Infection. Front. Immunol., 8, 246.

Preusse, M., K. Schughart, E. Wilk, F. Klawonn and F. Pessler, 2015: Hematological parameters in the early phase of influenza $A$ virus infection in differentially susceptible inbred mouse strains. $B M C$ research notes, 8, 225.

Ramis, A., G. van Amerongen, M. van de Bildt, L. Leijten, R. Vanderstichel, A. Osterhaus and T. Kuiken, 2014: Experimental infection of highly pathogenic avian influenza virus H5N1 in black-headed gulls (Chroicocephalus ridibundus). Vet. Res., 45, 84.

Ramsay, J. O., G. Hooker, D. Campbell and J. Cao, 2007: Parameter estimation for differential equations: a generalized smoothing approach. Journal of the Royal Statistical Society: Series B (Statistical Methodology), 69, 741-796.

REED, L. J. and H. MUENCH, 1938: A SIMPLE METHOD OF ESTIMATING FIFTY PER CENT ENDPOINTS. American Journal of Epidemiology, 27, 493-497.

Rhyoo, M. Y., K. H. Lee, O. K. Moon, W. H. Park, Y. C. Bae, J. Y. Jung, S. S. Yoon, H. R. Kim, M. H. Lee, E. J. Lee, M. R. Ki and K. S. Jeong, 2015: Analysis of signs and pathology of H5N1-infected ducks from the 2010-2011 Korean highly pathogenic avian influenza outbreak suggests the influence of age and management practices on severity of disease. Avian pathology : journal of the W.V.P.A, 44, 175-181.

S, N. M. a. K., 2000: Molecular Evolution and Phylogenetics. Oxford University Press, New York.

Saad, M. D., L. S. Ahmed, M. A. Gamal-Eldein, M. K. Fouda, F. Khalil, S. L. Yingst, M. A. Parker and M. R. Montevillel, 2007: Possible avian influenza (H5N1) from migratory bird, Egypt. Emerging infectious diseases, 13, 1120-1121.

Saitou, N. and M. Nei, 1987: The neighbor-joining method: a new method for reconstructing phylogenetic trees. Molecular biology and evolution, 4, 406-425.

Salaheldin, A. H., J. Veits, H. S. Abd El-Hamid, T. C. Harder, D. Devrishov, T. C. Mettenleiter, H. M. Hafez and E. M. Abdelwhab, 2017: Isolation and genetic characterization of a novel 2.2.1.2a H5N1 virus from a vaccinated meat-turkeys flock in Egypt. Virol J., 14, 48.

Salem, M. H. I., 2014a: Pathogenesis of highly pathogenic avian influenza virus in native ducks experimentally infected Master thesis, Faculty of Veterinary Medicine, Zagazig University. , 46, 50.

Salem, M. H. I., 2014b: Pathogenesis of highly pathogenic avian influenza virus in native ducks experimentally infected. Master thesis Department of Virology, Faculty of Veterinary Medicine, Zagazig University.

Sheta, B. M., T. L. Fuller, B. Larison, K. Y. Njabo, A. S. Ahmed, R. Harrigan, A. Chasar, S. Abdel Aziz, A. A. Khidr, M. M. Elbokl, L. Z. Habbak and T. B. Smith, 2014: Putative human and avian risk factors for avian influenza virus infections in backyard poultry in Egypt. Veterinary microbiology, 168, 208-213.

Smith, A. M., F. R. Adler and A. S. Perelson, 2010: An accurate two-phase approximate solution to an acute viral infection model. Journal of mathematical biology, 60, 711-726.

Smith, G. J., R. O. Donis, H. F. World Health Organization/World Organisation for Animal and H. E. W. G. Agriculture Organization, 2015: Nomenclature updates resulting from the evolution of avian influenza $\mathrm{A}(\mathrm{H} 5)$ virus clades 2.1.3.2a, 2.2.1, and 2.3.4 during 2013-2014. Influenza and other respiratory viruses, 9, 271-276.

Sturm-Ramirez, K. M., T. Ellis, B. Bousfield, L. Bissett, K. Dyrting, J. E. Rehg, L. Poon, Y. Guan, M. Peiris and R. G. Webster, 2004: Reemerging H5N1 influenza viruses in Hong Kong in 2002 are highly pathogenic to ducks. Journal of virology, 78, 4892-4901. 
Tamura, K., D. Peterson, N. Peterson, G. Stecher, M. Nei and S. Kumar, 2011: MEGA5: molecular evolutionary genetics analysis using maximum likelihood, evolutionary distance, and maximum parsimony methods. Molecular biology and evolution, 28, 2731-2739.

Tang, Y., P. Wu, D. Peng, X. Wang, H. Wan, P. Zhang, J. Long, W. Zhang, Y. Li, W. Wang, X. Zhang and X. Liu, 2009: Characterization of duck H5N1 influenza viruses with differing pathogenicity in mallard (Anas platyrhynchos) ducks. Avian pathology : journal of the W.V.P.A, 38, 457-467.

Van Campen, H., B. C. Easterday and V. S. Hinshaw, 1989: Destruction of lymphocytes by a virulent avian influenza A virus. J. Gen. Virol., 70 ( Pt 2), 467-472.

Vascellari, M., A. Granato, L. Trevisan, L. Basilicata, A. Toffan, A. Milani and F. Mutinelli, 2007: Pathologic findings of highly pathogenic avian influenza virus A/Duck/Vietnam/12/05 (H5N1) in experimentally infected pekin ducks, based on immunohistochemistry and in situ hybridization. Veterinary pathology, 44, 635-642.

Wasilenko, J. L., A. M. Arafa, A. A. Selim, M. K. Hassan, M. M. Aly, A. Ali, S. Nassif, E. Elebiary, A. Balish, A. Klimov, D. L. Suarez, D. E. Swayne and M. J. Pantin-Jackwood, 2011: Pathogenicity of two Egyptian H5N1 highly pathogenic avian influenza viruses in domestic ducks. Archives of virology, 156, 37-51.

Watanabe, Y., M. S. Ibrahim, H. F. Ellakany, N. Kawashita, T. Daidoji, T. Takagi, T. Yasunaga, T. Nakaya and K. Ikuta, 2012: Antigenic analysis of highly pathogenic avian influenza virus H5N1 sublineages co-circulating in Egypt. J. Gen. Virol., 93, 2215-2226.

Watanabe, Y., M. S. Ibrahim, H. F. Ellakany, N. Kawashita, R. Mizuike, H. Hiramatsu, N. Sriwilaijaroen, T. Takagi, Y. Suzuki and K. Ikuta, 2011: Acquisition of human-type receptor binding specificity by new H5N1 influenza virus sublineages during their emergence in birds in Egypt. PLoS pathogens, 7, e1002068.

Webster, R. G., W. J. Bean, O. T. Gorman, T. M. Chambers and Y. Kawaoka, 1992: Evolution and ecology of influenza A viruses. Microbiological reviews, 56, 152-179.

Wei, L., P. Jiao, Y. Song, L. Cao, R. Yuan, L. Gong, J. Cui, S. Zhang, W. Qi, S. Yang and M. Liao, 2013: Host immune responses of ducks infected with H5N1 highly pathogenic avian influenza viruses of different pathogenicities. Veterinary microbiology, 166, 386-393.

WHO/OIE/FAOH. N. Evolution Working Group WHO, O. a. F., 2012: Continued evolution of highly pathogenic avian influenza A (H5N1): updated nomenclature. Influenza and other respiratory viruses, 6, 1-5.

Wibawa, H., J. Bingham, H. Nuradji, S. Lowther, J. Payne, J. Harper, A. Junaidi, D. Middleton and J. Meers, 2014: Experimentally infected domestic ducks show efficient transmission of Indonesian H5N1 highly pathogenic avian influenza virus, but lack persistent viral shedding. PloS one, 9, 111.

Yamamoto, Y., K. Nakamura, K. Kitagawa, N. Ikenaga, M. Yamada, M. Mase and M. Narita, 2007: Severe nonpurulent encephalitis with mortality and feather lesions in call ducks (Anas platyrhyncha var. domestica) inoculated intravenously with H5N1 highly pathogenic avian influenza virus. Avian diseases, 51, 52-57.

Yen, H. L., J. R. Aldridge, A. C. Boon, N. A. Ilyushina, R. Salomon, D. J. Hulse-Post, H. Marjuki, J. Franks, D. A. Boltz, D. Bush, A. S. Lipatov, R. J. Webby, J. E. Rehg and R. G. Webster, 2009: Changes in H5N1 influenza virus hemagglutinin receptor binding domain affect systemic spread. Proceedings of the National Academy of Sciences of the United States of America, 106, 286-291.

Zhang, Y., B. D. Aevermann, T. K. Anderson, D. F. Burke, G. Dauphin, Z. Gu, S. He, S. Kumar, C. N. Larsen, A. J. Lee, X. Li, C. Macken, C. Mahaffey, B. E. Pickett, B. Reardon, T. Smith, L. Stewart, C. Suloway, G. Sun, L. Tong, A. L. Vincent, B. Walters, S. Zaremba, H. Zhao, L. Zhou, C. Zmasek, E. B. Klem and R. H. Scheuermann, 2017: Influenza Research Database: An integrated bioinformatics resource for influenza virus research. Nucleic Acids Res., 45, D466-d474. 
Table 1. Score describing clinical severity recorded from three randomly selected ducks per time point.

\begin{tabular}{|c|c|c|c|c|}
\hline $\begin{array}{c}\text { Time points } \\
\text { (hpi) }\end{array}$ & \multicolumn{3}{|c|}{ Clinical signs score } & $\begin{array}{c}\text { Total } \\
\text { score }\end{array}$ \\
\hline Mock & - & - & - & - \\
\hline 8 & + & - & - & 1 \\
\hline 16 & - & + & - & 1 \\
\hline 24 & + & + & + & 3 \\
\hline 32 & +++ & ++ & ++ & 7 \\
\hline 40 & + & + & - & 2 \\
\hline 48 & ++ & ++ & + & 5 \\
\hline 72 & +++ & +++ & +++ & 9 \\
\hline 96 & +++ & +++ & + & 7 \\
\hline 120 & +++ & ++ & ++ & 7 \\
\hline
\end{tabular}

- (no signs), + (nonspecific signs), ++ (specific signs) and +++ (severe neurological signs and paralysis) 


\section{Figure Legends}

Figure 1. Phylogenetic relation of A/chicken/Faquos/amn12/2011 to the Egyptian H5N1 clades. The analysis was based on partial nucleic acid sequence (1-384) of the HA segment. This HPAI H5N1 virus (indicated in the red box) clusters with the viruses within clade 2.2.1.2. The analysis was done using the BioEdit and MEGA 5 software tools. The evolutionary history of the virus was inferred using the neighbor-joining method. Phylogenetic relationships were estimated through a bootstrap trial of 1000 . The evolutionary distances were computed using the p-distance method. The numbers beside the nodes refer to the bootstrap value (node support value). The extent of the genetic changes (substitution per site) is indicated on a scale of 0.005 and can be used as a scale for the length of the branch inside the tree.

Figure 2. Weight loss and survival rates in mock and HPAI H5N1-infected ducks. A. Weight loss expressed as average weight (grams) \pm standard error of the mean (SEM) in mock-treated (dashed lines) and H5N1-infected (solid lines) ducks ( $n=3$ per group). $T=0$ indicates the weights before the experiment. B. Cumulative survival rates of H5N1-experimentally infected ducks recorded for a subgroup of 27 infected ducks. Ducks that succumbed to infection were counted as dead, and those that showed severe clinical signs such as high degree of paralyses or other neurological signs, had to be euthanized and were also counted as dead.

Figure 3. Selected post mortem lesions in HPAl H5N1-infected ducks. A. Congestion in internal organs of duck dead at $32 \mathrm{hpi}$. B. Liver of infected ducks at 32 hpi showing sub-mucosal hemorrhage (arrows). C. Petechial hemorrhage in pancreas (arrow) of ducks euthanized at $48 \mathrm{hpi}$. D. Heart of infected duck at $72 \mathrm{hpi} \mathrm{showing} \mathrm{focal}$ area of necrosis (arrow).

Figure 4. Selected histological findings in HPAI H5N1-infected (A-C) and mocktreated ducks (D-F). A. Lung of infected duck at 16 hpi showing hyperplasia of 
lymphoid tissue and mild lympho-histiocytic interstitial pneumonia. B. Brain of infected duck at $48 \mathrm{hpi}$ showing mild gliosis. C. Small intestine of infected ducks at $96 \mathrm{hpi}$ showing mild-to-moderate diffuse lympho-plasmacytic to heterophilic enteritis. D. Lung of mock-treated (uninfected) ducks showing moderate hyperemia. E. Brain of mocktreated ducks showing normal histology. F. Small intestine of mock-treated ducks showing normal histology. A, B, D, E: bar $=100 \mu \mathrm{m}$. C and F: bar $=200 \mu \mathrm{m}$

Figure 5. Changes in peripheral blood cells in H5N1-infected and mock-treated ducks. Blood was drawn from 3 ducks per time point. Panels show mean values $(+/-$ SEM) of absolute numbers of WBCs (A) and platelets (B), the percentages of lymphocytes (C), monocytes (D), granulocytes (E), heterophils (F), and reticulocytes (G). The percentages of these cells were calculated by dividing the absolute count of each cell by the WBC count. (H) Shows the granulocyte/lymphocyte ratio based on dividing absolute granulocyte counts by absolute lymphocyte counts. Solid lines refer to ducks infected with H5N1 virus. Dashed lines refer to mock-treated (uninfected) ducks. The differences between the mean values of each blood parameter in H5N1infected ducks and that of mock-treated ducks at the same time point were compared using Student's $t$ test (two-tailed and heteroschediastic unpaired). ${ }^{*}, P \leq 0.05 ;{ }^{* *}, P \leq$ $0.01 ;^{* * *}, P \leq 0.001 ;{ }^{* * * *}, P \leq 0.0001$. The analysis was done using GraphPad Prism V.5 software.

Figure 6. Relative quantification of HA MRNA of A/chicken/Faquos/amn12/2011(H5N1) and its transcription kinetics in various duck organs. HA mRNA of A/chicken/Faquos/amn12/2011(H5N1) was measured in various duck organs by RT-qPCR using the $2^{-\mathrm{DDCt}}$ method. The data are presented as mean $+/$ - SEM of fold change with respect to uninfected duck $(t=0 h)(n=3$ animals/time point). Asterisks indicate significance differences between mean viral HA mRNA RT-qPCR signal at each time point and the mean of the low background signal detected with these primers in mock-infected animals (around Ct 37-38). To compare replicate means, one way ANOVA followed by Tukey's multiple comparison test was 
conducted. *, $P \leq 0.05 ;{ }^{* *}, P \leq 0.01 ; * * *, P \leq 0.001$. The analysis was done using GraphPad Prism V.5 software. The $g$ value in each panel refers to exponential transcription rate of viral HA mRNA in the respective organ during the first $48 \mathrm{hpi}$. The transcription kinetics $(g)$ was calculated using the $\triangle \mathrm{Ct}$ values (HA mRNA normalized against $\beta$-actin mRNA) by fitting robust regression linear model using iterated reweighted least squares (IWLS) (Huber, 1981). Data were derived from the 3 mocktreated $(\mathrm{t}=0)$ animals and 3 animals at each of the indicated time points.

Figure 7. Correlation in HA mRNA transcription of A/chicken/Faquos/amn12/2011(H5N1) among selected organs across the time course. Numbers above the bars refer to the corresponding Pearson correlation coefficients $(r)$. Data were derived from the 3 mock-treated $(t=0)$ animals and 3 H5N1infected animals at each of the indicated time points. Pearson correlation coefficient was calculated using $R$ program $V$. 3.0.2.

\section{Supplementary files}

Table S1. Viral strains belonging to various clades used to generate the phylogenetic analysis. HA mRNA accession numbers are according to NCBI. The strain that is the subject of this study (A/chicken/Faquos/amn12/2011) is printed in red.

Table S2. Comparison of selected amino acid residues in the HA protein in the isolates belonging to clade 2.2.1.2 and that of clades $(2.2,2.2 .1,2.2 .1 .1$ and 2.2.1.1). The right hand column lists amino acids conserved among the analyzed clade 2.2.1.2 strains, including $\mathrm{A} /$ chicken/Faquos/amn12/2011. A $\Delta$ denotes a deletion. The capital letters refer to the one-letter amino acid abbreviations. 
Figure S1. Alignment of amino acid residues (110-165) of the HA protein of Egyptian H5N1 clades. The name and aa sequences of $\mathrm{A} /$ chicken/Faquos/amn12/2011 are printed in red and show an aa signature identical to that of the viruses belonging to clade 2.2.1.2. Protein IDs and amino acid sequences are according to the US National Center for Biotechnology Information (NCBI). A dash (-) in the alignment denotes a deletion. The alignment was done by the ClustalW method using MEGA 5.0 software (http://mega.software.informer.com/5.0/). 